\title{
STEIN MANIFOLDS ON WHICH THE STRONG POINCARÉ PROBLEM CAN BE SOLVED
}

\author{
ROBERT EPHRAIM
}

\begin{abstract}
Let $M$ be a Stein manifold. Suppose every meromorphic function on $M$ may be written as the quotient of two holomorphic functions which are pointwise relatively prime at every point of $M$. Then it will be shown that $H^{2}(M, Z)=0$. Thus, the solvability of the Strong Poincare Problem is equivalent to the vanishing of the second integral cohomology, which in turn is equivalent to the solvability of Cousin II, all on a Stein manifold $\boldsymbol{M}$. This closes a gap in the classically known theory.
\end{abstract}

Let $M$ be a complex manifold. The Strong Poincaré Problem asks whether every meromorphic function on $M$ can be written as the quotient of two holomorphic functions on $M$ which are pointwise relatively prime at every point of $M$. If $M$ is a Stein manifold, then it is well known that a sufficient condition for the Strong Poincaré Problem to be solvable is given by $H^{2}(M$, $Z)=0$. This is an immediate consequence of Cartan's Theorems A and B, some standard exact sequences, and the fact that every divisor on $M$ is the difference of two positive divisors. The question of finding necessary conditions for the solution of the Strong Poincare Problem on Stein manifolds has been ignored, perhaps because the same exact sequences fail to give a quick answer. I will show:

TheOREM. Let $M$ be a Stein manifold on which the Strong Poincaré Problem can be solved. Then $H^{2}(M, Z)=0$.

Thus, for a Stein manifold $M$, the solvability of the Strong Poincaré Problem is equivalent to $H^{2}(M, Z)=0$ which in turn is equivalent to the solvability of Cousin II. This fills the gap in the classical theory. (See [2] for an account of that classical theory.) Let me note that Dales [1] has proven an analogue of this result for Stein compacts $K \subset M$ for which the ring of germs of functions holomorphic on a neighborhood of $K$ is a noetherian ring. His proof depended heavily on the noetherianness and cannot be used in the manifold case.

The proof will use the following

LemMa. Let $M$ be a Stein manifold, let $\left\{H_{i}\right\}_{i \in I}$ be a locally finite family of

Received by the editors September 15, 1977.

AMS (MOS) subject classifications (1970). Primary 32E10; Secondary 32A20.

Key words and phrases. Stein manifold, Strong Poincaré Problem, meromorphic function, positive divisors, Cousin II, integral cohomology. 
irreducible hypersurfaces, and for each $i \in I$, let $\nu_{i} \geqslant 0$ be an integer. Then there is a holomorphic function $f$ on $M$ which for each $i \in I$ vanishes on $H_{i}$ precisely to order $\nu_{i}$. (It may, of course, vanish elsewhere as well.)

Proof. Since the $H_{i}$ are locally finite we may define a coherent sheaf of ideals $\mathcal{G}$ by $\mathcal{F}=$ sheaf of germs of holomorphic functions vanishing on $H_{i}$ to order at least $\nu_{i}$ for all $i \in I$.

The local finiteness of the $H_{i}$ also shows that $H=\cup H_{i}$ is a hypersurface in $M$ whose global irreducible components are just the $H_{i}$. For each $i \in I$ choose a point $x_{i} \in H_{i} \cap \operatorname{Reg}(H)$. Then $X=\left\{x_{i}\right\}_{i \in I}$ is a discrete closed subset of $M$. Thus $X$ is an analytic subset of $M$, and $\mathfrak{n}=$ sheaf of germs of holomorphic functions vanishing on $X$ is a coherent sheaf of ideals defining the analytic set $X \subset M$.

Then for $x \notin X$ we clearly have $g_{x}=(\mathfrak{n} \cdot g)_{x}$. And, it follows from Nakayama's lemma that for $x \in X$ we have $g_{x} \neq(\mathfrak{n} \cdot g)_{x}$. Thus $(g / n \cdot g)_{x}$ $\neq 0$ if and only if $x \in X$. Since $X$ is a discrete closed subset of $M$, it follows that we may find a section $\delta \in H^{0}(M, g / \mathfrak{n} \cdot \mathcal{g})$ for which the germs $\delta_{x} \neq 0$ for any $x \in X$.

By Cartan's Theorem B we may find a section $f \in H^{0}(M, g)$ whose image in $H^{0}(M, g / \mathfrak{n} \cdot \mathscr{G})$ equals $\mathcal{S}$. But then $f$ is a holomorphic function which vanishes to order at least $\nu_{i}$ on $H_{i}$ for all $i \in I$. Since $\mathcal{S}_{x} \neq 0$ for all $x \in X$, it follows that $f_{x} \notin(n \cdot g)_{x}$ for all $x \in X$. Setting $x=x_{i}$ this shows that $f$ vanishes to order at most $\nu_{i}$ on $H_{i}$. Since this is true for any $i \in I, f$ is the function sought.

Theorem. Let $M$ be a Stein manifold on which the Strong Poincaré Problem can be solved. Then $H^{2}(M, Z)=0$.

Proof. By the equivalence of the solvability of Cousin II and of $H^{2}(M$, $Z$ ) $=0$ we need only show that every divisor is the divisor of a meromorphic function on $M$. Since every divisor on $M$ may be written as the difference of two positive divisors, it suffices to show that every positive divisor is the divisor of a holomorphic function on $M$.

Let $D$ be a positive divisor on $M$. We may write $D=\Sigma \nu_{i}\left[D_{i}\right]$, where each $\left[D_{i}\right]$ is the divisor associated to the irreducible hypersurface $D_{i}$, and where each $\nu_{i}$ is a positive integer. Applying the lemma we may find a holomorphic function $f$ vanishing on each $D_{i}$ precisely to order $\nu_{i}$. Then, denoting the divisor of $f$ by $\operatorname{div}(f)$, we have $\operatorname{div}(f)=\sum \nu_{i}\left[D_{i}\right]+\sum \mu_{j}\left[E_{j}\right]$, where each $E_{j}$ is an irreducible hypersurface and each $\mu_{j}$ is a positive integer. Furthermore, $D_{i} \neq E_{j}$ for any $i$ and $j$.

Again applying the lemma we may find a holomorphic function $g$ which vanishes on each $E_{j}$ precisely to order $\mu_{j}$ and which vanishes on each $D_{i}$ precisely to order 0 (that is, it does not vanish on any $D_{i}$ ). Then $\operatorname{div}(g)=$ $\sum \mu_{j}\left[E_{j}\right]+\sum \gamma_{k}\left[H_{k}\right]$, where each $H_{k}$ is an irreducible hypersurface and each $\gamma_{k}$ is a positive integer. Also, $D_{i} \neq H_{k}$ for any $i$ and $k$.

Then $f / g$ is a meromorphic function for which $\operatorname{div}(f / g)=\sum \nu_{i}\left[D_{i}\right]-$ 
$\sum \gamma_{k}\left[H_{k}\right]$. By assumption we can find two holomorphic functions $F$ and $G$ which are pointwise relatively prime at every point of $M$ and which satisfy $f / g=F / G$. But then we immediately get $\operatorname{div}(F)=\Sigma \nu_{i}\left[D_{i}\right]=D$. Thus the arbitrarily chosen positive divisor $D$ is the divisor of the holomorphic function $F$; so we are done.

\section{REFERENCES}

1. H. G. Dales, The ring of holomorphic functions on a Stein compact set as a unique factorization domain, Proc. Amer. Math. Soc. 44 (1974), 88-92.

2. L. Hormander, An introduction to complex analysis in several variables, Van Nostrand, Princeton, N. J., 1966.

Department of Mathematics, Herbert H. Lehman College, CUNY, New York, New YORK 10468 